\title{
Outcome of Early Removal of Urinary Catheter after Transurethral Resection of Prostate (TUR-P)
}

\author{
H. Mazahir Zulfiqar, Sajjad Ahmed, Zein UL Amir, M. Afzal Farooqui
}

\section{ABSTRACT:}

Objective: To determine the outcome of early removal of urinary catheter, and predict the possibility of TUR-P as a day care surgery, in terms of reduced hospital stay.

Study Design \& Setting: It was a cross sectional study design with non-probability sampling conducted at Department of Urology, Rawalpindi medical college from January to July 2017.

Methodology: Total 190 patients fulfilled inclusion and exclusion criteria were selected for the study. Patient underwent TUR-P followed by catheter irrigation. Time was noted as 'zero' hour. The color of the effluent was grossly monitored. When the effluent became clear the catheter was removed and time noted. After successful voiding, patient was discharged and time noted. Success is if the duration from time 'zero' hours to catheter removal is within 24 hours and duration of hospital stay is within 36 hours. A time line greater is considered to be failure. Data analysis was done using SPSS version 15.0. For quantitative variables like age, time of catherization and hospital stay, median and standard deviation was calculated. For qualitative variables like success, frequency and percentages were calculated. Groups and tables were made to present the data.

Results: Mean duration of catherization after TUR-P is $9.67 \pm 2.36$ (hrs. min). Hospital stay was $26.73 \pm 6.24$ hours. Frequency and percentage of successful outcome was 152 out of 190 patients and 82.6\% respectively.

Conclusion: Our study favors that in selected patients TUR-P can be performed with shorter hospital stay with minimal postoperative complications. This will benefit the patient in terms of reducing comorbids and considerable departmental financial savings.

Key Words: Trans-urethral resection of Prostate, Benign Prostatic Hyperplasia

How to cite this Article:

Zulfiqar HM, Ahmed S, UlAmir Z, Farooqi MA. Outcome of Early Removal of Urinary Catheter after Transurethral Resection of Prostate (TUR-P). J Bahria Uni Med Dental Coll. 2021; 11(3):DOI: https://doi.org/10.51985/GFXM9719

This is an Open Access article distributed under the terms of the Creative Commons Attriution Non Commercial Liciense (http:// creativecommons/org/licences/by-nc/4.0) which permits unrestricted non commercial use, distribution and reproduction in any medium, provided the original work is properly cited.

INTRODUCTION:

The gold standard treatment for obstructing enlargement of the prostate is Transurethral Resection of Prostate (TURP). ${ }^{1}$ It provides the long-term benefit of improved voiding scores and variables ${ }^{2}$ with reduced morbidity. ${ }^{3}$ Enlarged prostate is a disease of older men. ${ }^{4}$ Variables such as various co-morbidities and co-morbids as a result of the disease itself, such as urinary tract infections, effect of prolonged

г- - - - - - - - - - - - -

H. Mazahir Zulfiqar

Senior Lecturer, Department of Urology

I Rawalpindi Medical University

Email: drmazahir2005@gmail.com

I Sajjad Ahmed

Senior Registrar, Department of Urology

I Rawalpindi Medical University

Zein UL Amir

Professor, Department of Urology

I Rawalpindi Medical University

M. Afzal Farooqui (Late)

I Professor, Department of Urology

I Rawalpindi Medical University

I Received: 21-04-2021

Accepted: 30-06-2021

1 Accpted. $3006-2021$ catherization, prolonged hospital stay add to the morbidity of such patients. ${ }^{5}$ Attempt in modifying variables associated with the disease process can reduce the morbidity of the patient.

The management of patients with enlarged prostate is dependent on the severity of the symptoms translated by the IPSS. ${ }^{6}$ A mild score warrants watchful waiting with lifestyle modifications. ${ }^{7,8}$ Moderate symptoms warrant the use of medical therapy and / or a combination of medical therapy options. ${ }^{9,10}$ Severe symptoms, failure of medical therapy or developing complications as a result of prolonged obstruction warrants surgical intervention. Despite the availability of many minimal invasive surgical modalities, TUR-P remains the gold standard. ${ }^{11}$

Study conducted by Cheuk Fan Shum et al and Prasopsuk S. et al, proves that removal of the catheter on the first post operative day had a success of $98 \%$ with significant reduction in complications. ${ }^{12,13} \mathrm{~J}$. Chander et al went further to remove the catheter on the same operative day after TUR-P without significant complications. ${ }^{3}$

TUR-P is an endoscopic modality that resects the prostatic lobes using either mono-polar or bi-polar current across a 
resecting loop, using suitable fluid as irrigation. ${ }^{11,14}$ Post operatively; the effects of TUR-P can be assessed by follow up IPSS and uroflowmetry. ${ }^{2,15}$

In this study setting; the protocol is patient after TUR-P is observed for a minimum of 5 days in hospital. On the fifth post-operative day the patient's catheter is removed and he is discharged after a successful trial of voiding per urethra. Due to scarce resources and a high volume of waiting patients for TUR-P this study addressed the impact of same day catheter removal after TUR-P with quality care and SOPs and its effects on hospital stay. This in turn is an attempt to decreased morbidity due to prolonged catherization, reduced hospital stay, waiting time and have less financial burden on patients and indeed was the rationale of the study. Hence; this study was aimed to determine the outcome of same day removal of urinary catheter after TUR-P, and predict the possibility of TUR-P as a day care surgery, in terms of reduced hospital stay.

\section{METHODOLOGY:}

It was a cross sectional study, conducted in the department of Urology and transplantation, Rawalpindi university medical college, from January to July 2017. One hundred and ninety patients were selected for the study using the WHO calculator. The variables used in the calculator were: confidence level $95 \%$, anticipated populated proportion $98 \%$ ${ }^{3}$, absolute precision required $2 \%$. Non-probability sampling technique was used for patient selection. The inclusion criteria is, age greater than 40 years but less than 75 years $^{16}$, indications to proceed towards TUR- $\mathrm{P}^{17}$, and prostate volume less than 50 grams on trans-abdominal ultrasound. Factors such as carcinoma of the prostate, hypertension, diabetes, and significant intra-operative complications such as urethral injury, bladder perforation or uncontrolled hematuria were excluded from the study.

Approval of the ethical committee was taken. Patients fulfilling the inclusion criteria were counseled and informed consent was taken. All patients selected for the study under went comprehensive evaluation including a complete history with evaluation of the international prostatic symptoms score (IPSS), clinical examination and basic laboratory and radiological investigations. ${ }^{18}$ Ensuring fitness for spinal anesthesia and a negative urine culture, the patient was dated for TUR-P. After overnight admission, patient underwent conventional TUR-P. ${ }^{14}$ A 24 Fr. 30 degrees, mono-polar, continuous flow, resectoscope with non-revolving sheath was used in all cases. Total procedure time did not exceed 60 minutes. After satisfactory resection, meticulous hemostasis was done and finally a check cystoscopy performed to ensure no residual prostatic chip remained in the bladder. At the end of the procedure a $22 \mathrm{Fr}$. three way silicone coated irrigation urethral catheter was passed and the balloon inflated, irrigation with $0.9 \%$ saline was started. This was noted to time 'Zero' hours.
In the post operative period pulse, blood pressure, temperature and oxygen saturation of the patient were monitored. Urine output per catheter was measured hourly by the formula of deducting the volume of urine output per catheter from the total volume of saline delivered as bladder irrigation. The colour of the effluent was grossly monitored by the naked eye till it became clear and transparent. At that time the irrigation is withheld for 1 hour, after which if the effluent remained clear the irrigation was stopped and the urethral catheter is removed. If the effluent does not remain clear, irrigation is resumed again.

After removal of the catheter, the patent was allowed to void per urethra. Symptom score (IPSS), hematuria and patient comfort on voiding are addressed. If all is well, the patient is discharged to follow up. Success is define as the duration from time 'zero' hours to catheter removal is less than 24 hours and the duration of hospital stay is less than 36 hours. A time line greater than expressed is considered to be failure.

Data analysis was done using SPSS version 15.0. For quantitative variables like age, time of catherization and hospital stay, median and standard deviation was calculated. For qualitative variables like success, frequency and percentages were calculated. Groups and tables were made to present the data. P-value $<0.05$ was considered as statistically significant.

\section{RESULTS:}

A total of 190 patients were enrolled in this study. Descriptive statistics of age (years) of the patient was calculated in terms of mean and standard deviation, tabulated in table 1 . The time (hours. minutes) from time 'zero' to catheter removal was calculated in terms of mean and standard deviation. Mean time is $9.67 \pm 2.36$ ( $\mathrm{hrs}$. min). The post catheter removal IPSS showed a mean score of $34.69 \pm 1.34$. Hospital stay was measured in terms of hours. The mean hospital stay was $26.73 \pm 6.24$ hours. Success is defined as the time in hours, passed for catheter removal after 'zero' hour to be within 24 hours and satisfactory discharge from hospital within 36 hours. In our study, frequency and percentage of successful outcome were 152 out of 190 patients and $82.6 \%$ respectively. This is shown in graph 1 . Effect modifiers like age were controlled by stratification, which was compared with the successful outcome in the study. Chi - square test was used and showed $\mathrm{P}$ value of 0.57 , which was statistically not significant. This is shown in table 2 .

\section{DISCUSSION:}

The prostatic gland lies between the two urinary sphincters, the bladder neck superiorly and the voluntary striated sphincter inferiorly. The prostate surrounds the prostatic part of the urethra. The gland is composed of zones; peripheral, central, transitional and fibromuscular stroma ${ }^{19}$ Cystoscopically, the prostate can be viewed to have 2 lateral lobes and a median lobe. ${ }^{20}$ Hyperplasia of the lobes leads to obstruction in the urinary flow that can progress to acute 
Table 1:

\begin{tabular}{|l|c|c|c|c|c|}
\hline \multicolumn{1}{|c|}{ Variables } & N & Minimum & Maximum & Mean & $\begin{array}{c}\text { Standard } \\
\text { Deviation }\end{array}$ \\
\hline Age (yrs) & 190 & 41 & 75 & 64.4 & 7.35 \\
\hline Time to removal of catheter (Hr. Min.) & 190 & 6.20 & 23.0 & 10.7 & 2.36 \\
\hline IPSS score, post TUR-P, post catheter removal & 190 & 27 & 35 & 34.69 & 1.342 \\
\hline Hospital stay, post TUR-P. (Hr. Min.) & 190 & 23.30 & 47.0 & 26.7 & 6.24 \\
\hline
\end{tabular}

Graph 1: Frequency and percentage of Success.

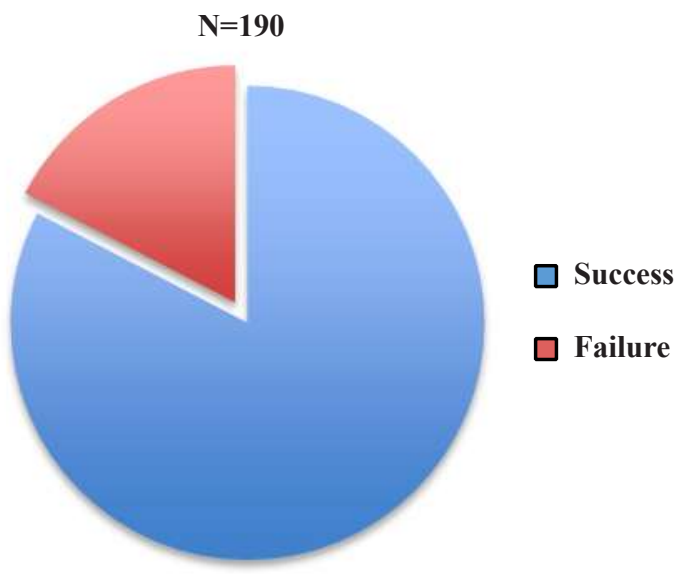

Table 2: Comparison of Age stratification with the success of TURP in the study

\begin{tabular}{|c|c|c|c|c|}
\hline & \multicolumn{2}{|c|}{ Success } & \multirow{2}{*}{ P-value } \\
\hline & & Yes & No & \\
\hline \multirow{6}{*}{ Age groups } & \multirow{2}{*}{$<=60 \mathrm{yrs}$} & 43 & 12 & \multirow{8}{*}{0.576} \\
\hline & & $27.4 \%$ & $36.4 \%$ & \\
\hline & \multirow{2}{*}{61 - 70 yrs. } & 79 & 15 & \\
\hline & & $50.3 \%$ & $45.5 \%$ & \\
\hline & \multirow{2}{*}{ above 70 yrs. } & 35 & 6 & \\
\hline & & $22.3 \%$ & $18.2 \%$ & \\
\hline \multirow{2}{*}{\multicolumn{2}{|c|}{ Total }} & 157 & 33 & \\
\hline & & $100.0 \%$ & $100.0 \%$ & \\
\hline
\end{tabular}

or chronic urinary retention. ${ }^{21}$ These obstructive symptoms can be translated by the patient via the IPSS score. ${ }^{22}$

In our study, the mean duration of urethral catheter removal is $10.7 \mathrm{hrs} / \mathrm{min}$ and theses result are in accordance with the prospective study conducted by Cheuk Fam Shum et al on 200 patients undergoing TUR-P, reported that 156 patients (78\%) had successful outcome after catheter removal on the first postoperative day. ${ }^{13}$ Another study by J. Chander et al showed that the mean duration of catheterization was 6.54 hours. However, 10 patients required re-catherization. ${ }^{3}$

As per our current departmental practice, the duration of hospital stay is five days or 120 hours. In our study the hospital stay has significantly reduced to a mean on 26 hours. J. Chander et al demonstrated in his study that by earlier catheter removal, duration of hospital stay had reduced from 3.1 to 1.28 days. ${ }^{3}$ Similar results were seen in the study by Cheuk Fam Shum et al in which overall hospital stay was 1.6 days. ${ }^{13}$

The post TUR-P, post catheter removal, IPSS in our study is severe. The mean IPSS was $34.69 \pm 1.34$ with a p-value of 0.710 . Comparing with the study of Bae WJ et al, their mean IPSS was $21.45 \pm 0.54^{23}$; it is believed that severe IPSS was because of the severe dysuria felt by the patient in their earlier voids. Despite an improved caliber of urinary stream the patient felt hesitant to void post early catheter removal.

In our study; successful outcome was seen in 157 patients $(82.6 \%)$, while failed outcome was seen in 33 patients (17.4\%). This result is favorable towards our attempt of early catheter removal after TUR-P in selected patients. J. Chander et al show similar results in which $98 \%$ of their 64 patients were discharged within 23 hours. They concluded that TUR-P could be safely conducted as a day care surgery. ${ }^{3}$ However, their population was 64 patients while our study had a larger cohort of 190 patients.

Cheuk Fan Shum et al concluded in their study of 40 patients, that catheter free first postoperative day discharge was safe. However this study had a small population of 40 patients and their energy source for TUR-P was bi-polar while we used mono-polar. ${ }^{13}$

Okeke LI in his study day care transurethral prostatectomy in Nigeria ${ }^{15}$ evaluated 180 patients after TUR-P and did not insert a urethral catheter post operatively. They concluded that TUR-P is a safe procedure to be conducted as a day case surgery. However choose to keep the patient on catheter irrigation in the immediate postoperative period in lieu of good and safe surgical practice.

The study by Prasopsuk S. et al also favors our findings towards early catheter removal after TUR-P. ${ }^{12}$ Despite a result that is favorable to our attempt to decrease hospital stay while maintaining good practice standards and safety of our patients, our study has some limitations. Long term follow up in term of IPSS and uroflowmetry is lacking. This is because our patients are financially strained and arrive at our center after travelling long distances from their homes, hence frequent follow up becomes unaffordable for most of the patients. Uroflowmetry is a qualitative adjunct that adds to the value of IPSS, we could not perform as we did not 
have a uroflowmeter, and provision by the government is awaited. Screening patients for their pre-operative PSA value to rule out suspicion towards carcinoma of the prostate is not performed in this study as it was not part of our departmental protocol to screen all patients. Only those with symptoms and clinically hard prostate on digital rectal examination were screened.

\section{CONCLUSION:}

In selected patients TUR-P can be performed with shorter hospital stay with minimal postoperative complications. This effort will help us to reduce, the patient waiting time to TUR-P, the complications associated with prolonged catherization and reduce the expense per patient.

Authors Contribution:
H. Mazahir Zulfiqar: Primary for data collection and writing
Sajjad Ahmed: Data Collection
Zein Ul Ameer: Primary Surgeon
M. Afzal Farroqui: Consultation and Guidance towards
objective

\section{REFERENCES:}

1. Pujari NR. Transurethral Resection of Prostate is Still the Gold Standard for Small to Moderate Sized Prostates. J Integr Nephrol Androl 2016;3:68-9.

2. Wada N, Kikuchi D, Tateoka J, Abe N, Banjo H, Tsuchida M, Hori J, Tamaki G, Kita M, Kakizaki H. Long-term symptomatic outcome after transurethral resection of the prostate: A urodynamics-based assessment. International Journal of Urology. 2019;26(11):1071-5.

3. J. Chander, V. Vanitha, P. Lal, V.K. Ramteke. Transurethral resection of the prostate as catheter-free day-care surgery, BJUI 2003; 94:422-425.

4. Lokeshwar SD, Harper BT, Webb E, Jordan A, Dykes TA, Neal Jr DE, Terris MK, Klaassen Z. Epidemiology and treatment modalities for the management of benign prostatic hyperplasia. Translational andrology and urology. 2019;8(5):529.

5. Nakagawa T, Toguri AG: Early catheter removal following transurethral prostatectomy: A study of 431 patients. Med Princ Pract 2006; 15: 126-130.

6. Wang JY, Liu M, Zhang YG. Relationship between lower urinary tract symptoms and objective measures of benign prostatic hyperplasia: a Chinese survey. Chin Med J (Engl). 2008;121(20):2042-5.

7. Kerna NA, Nwokorie U, Flores JV, Holets HM, Akabike LU, Chen MH, Solomon EO, Pruitt KD, Waugh S. Benign Prostatic Hyperplasia (BPH): Perennial Plague of the Prostate. EC Clinical and Medical Case Reports. 2021;4:53-61.

8. Langan RC. Men's Health: Benign Prostatic Hyperplasia. FP essentials. 2021;503:18-22.
9. Roehrborn CG, Siami P, Barkin J, Damião R, Major-Walker $\mathrm{K}$, Morrill B. The effects of dutasteride, tamsulosin and combination therapy on lower urinary tract symptoms in men with benign prostatic hyperplasia and prostatic enlargement: 2 -year results from the CombAT study. J Urol. Feb 2008;179(2):616-21; discussion 621.

10. Sterling J, Farber N, Gupta NK. Comparing outcomes of medical management and minimally invasive surgical techniques for lower urinary tract symptoms due to BPH. Current urology reports. 2019;20(6):1-7.

11. Burke N, Whelan JP, Goeree L, Hopkins RB, Campbell K, Goeree R, et al. Systematic review and meta-analysis of transurethral resection of the prostate versus minimally invasive procedures for the treatment of benign prostatic obstruction. Urology. 2010;75(5):1015-22

12. Prasopsuk S, Tunruttanakul S. Safety of a first-day catheter removal after transurethral resection of the prostate (TURP): a propensity score-matched historical control study. Insight Urology. 2021;42(1):40-5.

13. Cheuk Fan Shum, Amit Mukherjee, Chang Peng Colin Teo. Catheter-free discharge on first postoperative day after bipolar transurethral resection of prostate: Clinical outcomes of 100 cases, International Journal of Urology 2013; 20(12).

14. Shrotri NC. How to Perform a Good TURP. In Practical Tips in Urology 2017 (pp. 487-491). Springer, London.

15. Okeke LI. Day case transurethral prostatectomy in Nigeria. West Afr J Med. 2004 Apr-Jun;23(2):128-30.

16. Lim KB. Epidemiology of clinical benign prostatic hyperplasia. Asian journal of urology. 2017 Jul 1;4(3):148-51.

17. Strebel RT, Kaplan SA. The state of TURP through a historical lens. World Journal of Urology. 2021 Mar 27:1-8.

18. Braeckman J, Denis L. Management of BPH then 2000 and now 2016-From BPH to BPO. Asian journal of urology. 2017 Jul 1;4(3):138-47.

19. Laucirica O, Catalá V, Vilanova JC. Anatomy of the prostate. In Atlas of Multiparametric Prostate MRI 2018 (pp. 23-46). Springer, Cham.

20. Goddard JC. The history of the prostate, part one: say what you see. Trends in Urology \& Men's Health. 2019;10(1):2830.

21. Descazeaud A, Robert G, de La Taille A. Management of the bladder outlet obstruction associated with $\mathrm{BPH}$ in patients with special circumstances and/or complications. Progres en Urologie: Journal de L'association Francaise D'urologie et de la Societe Francaise D'urologie. 2018;28(15):868-74.

22. D'Agate S, Wilson T, Adalig B, Manyak M, Palacios-Moreno JM, Chavan C, Oelke M, Roehrborn C, Della Pasqua O. Impact of disease progression on individual IPSS trajectories and consequences of immediate versus delayed start of treatment in patients with moderate or severe LUTS associated with BPH. World journal of urology. 2020;38(2):463-72.

23. Bae WJ, Ahn SG, Bang JH, Bae JH, Choi YS, Kim SJ et al. Risk Factors for Failure of Early Catheter Removal After Greenlight HPS Laser Photoselective Vaporization Prostatectomy in Men With Benign Prostatic Hyperplasia. Korean J Urol. 2013;54(1):31-5. 